\section{An Introduction to the Helium Ion Microscope}

John Morgan, John Notte, Raymond Hill, Bill Ward ALIS Corporation, Peabody, MA

jmorgan@aliscorporation.com

\section{Introduction}

In order to get high resolution images from any scanning beam microscope one must be able to produce a sufficiently small probe, have a small interaction volume in the substrate and have an abundance of information-rich particles to collect to create the image. A typical scanning electron microscope is able to meet all of these requirements to some degree. However, a helium ion microscope based on a Gas Field Ion Source (GFIS) has significant advantages over the SEM in all three categories.

The ultimate probe size in a SEM is limited by diffraction and chromatic aberration. Due to the very high source brightness and the shorter wavelength of the helium ions, it is possible to focus the ion beam to a smaller probe size relative to the SEM.

An electron beam has a relatively large excitation volume in the substrate. This limits the resolution of an SEM regardless of the probe size. A helium ion beam does not suffer from this effect as the excitation volume is much smaller than that of the SEM.

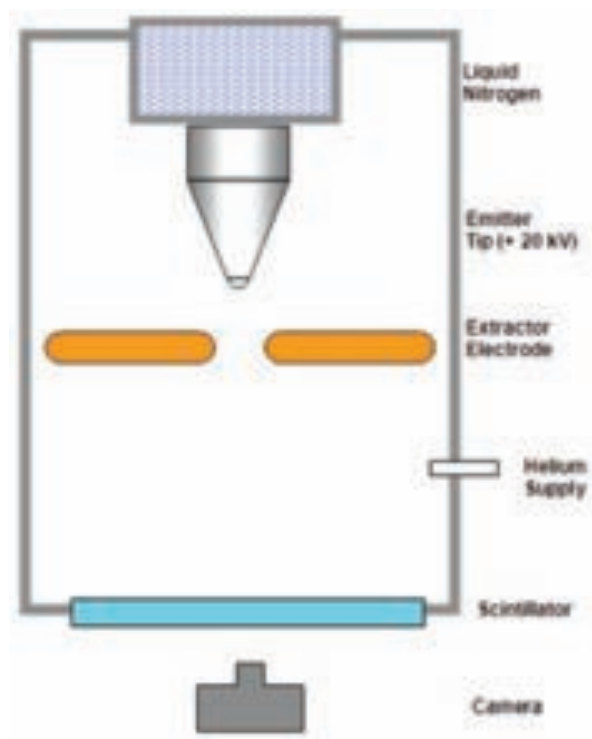

Figure 1: The geometry of a simple Field Ion Microscope (FIM).
SEM's are typically run at or near their secondary electron unity crossover point to minimize charging of the sample. This implies that for each incoming electron, one secondary electron is made available for imaging. The situation with the helium ion beam is much more favorable. Typically for each incoming helium ion from 2 to 8 secondary electrons are generated. This abundance of secondary electrons allows for very high contrast imaging.

In addition to secondary electrons, backscattered helium ions are also available for imaging. These ions are not as abundant as secondary electrons, but do provide unique contrast mechanisms that allow quantitative discrimination between materials with submicron spatial resolution.

\section{The Ion Source}

In recent decades there have been many efforts to develop a high brightness source of noble gas atoms with a low energy spread. A recent summary of these efforts, and their limitations, can be found in the literature [1]. The properties of high brightness and low energy spread are desired so that the beam of ions can be focused to the smallest possible probe size on the sample. A beam consisting of noble gas ions is preferred to minimize any chemical, electrical, or optical alteration of the sample. If helium is used as the noble gas, there is the additional benefit of minimal sputtering of the substrate.

ALIS Corporation has developed a high brightness, monochromatic source of noble gas ions, which is well-suited to high resolution microscopy. The ion source developed at ALIS Corporation is best understood by comparison with a related technology, the Field Ion Microscope (FIM).

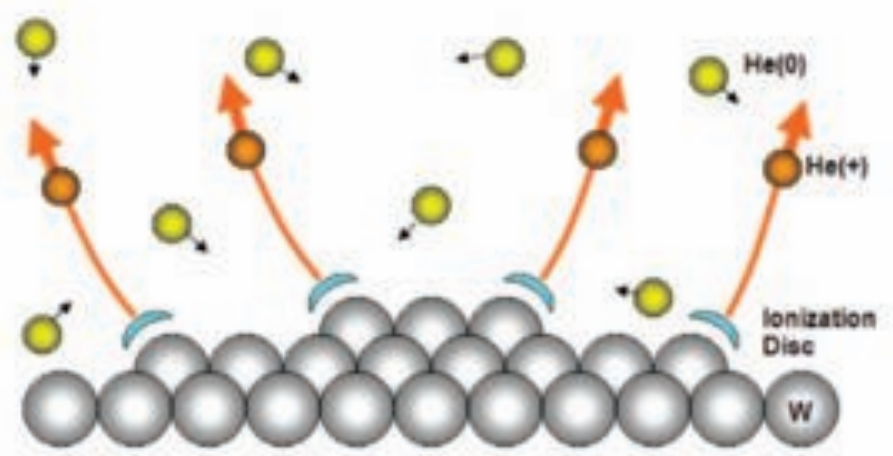

Figure 2: Neutral helium atoms (yellow) are drawn towards the tip by a polarization effect. When they pass through the ionization disk (blue) they are ionized (orange) and are accelerated away from the tip.

\section{A Closely Related Technology: The Field Ion Microscope}

The field ion microscope was optimized in 1955 by E. Müller, and K. Bahadur. The concept relies upon a cryogenically cooled, sharp tip in a UHV vacuum system, to which small amounts of helium gas have been admitted. In its simplest form, the geometry is shown in figure 1 .

The sharp tip was originally made of tungsten, and the sharp tip is commonly achieved by standard electro-chemical etching procedures. When the tip is positively biased in the presence of an adjacent grounded electrode, a very large electric field is formed at the tip. The maximum field is, of course, at the sharpest corners, and with only modest voltages $(5 \mathrm{kV}$ to $30 \mathrm{kV})$ field strengths of 5 $\mathrm{V} / \AA ̊ \AA$ can be achieved. At this field strength, the tungsten atoms from the most protruding points will be field evaporated from the bulk. By this process, the end shape is significantly smoothed until the sharpest corners reside at steps of the crystal planes. At a reduced voltage, corresponding to a field strength of $3 \mathrm{~V} / \AA$, the tungsten will not be field evaporated, but any neutral gas atoms in the vicinity of the sharpest corners will be ionized by the process of electron tunneling. This

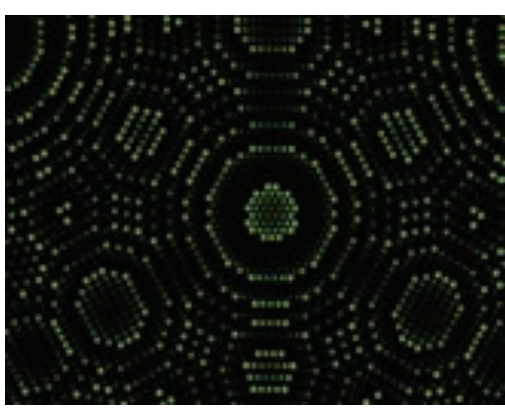

Figure 3: A typical FIM image shows many helium beams. Each beam emanates from an ionization disc above the most protruding atoms. ionization region is disc shaped with a diameter of just a few $\AA$, and a thickness of approximately $0.25 \AA$. The resulting positive ion is immediately accelerated away from the tip. Each such corner atom thus produces a beam that may be imaged by allowing it to strike a scintillator. Several ionization disks are shown in figure 2. The corresponding FIM image is shown in figure 3. 


\section{Multiphase Materials Characterization \\ Fully Integrated...Totally Seamless}

Powerful Analytical Tools Optimized for Your SEM to Provide Results with Confidence

- Pegasus . Simultaneous daka acquisitions for EDS (chemistry) and EDSO (erystallography)Includes the patenied Chi-Scan sottware to improve multiphase analysis capabitity

- Neptune..Complimentary uniee ef tos and WDS for superier X-eay mieroanalysils-imeroves quantification especially for low level concentrations

* Trident..utimabe materials characterkation system integrabes EDS, EESD, and WDS with no analytical performance compromise-Prowibles complete solusions for all your masarials character. iation requicements

Results with Confidence

As twe world's leader in Electron Beam Micreanalysis. EDWX continues to mise the performance standard with innovative features that ase easy to adapt and provides results that you can bust to be complete and accurise.

For move information on our Seamless Integrated Systems for EDS, EBSD, and WDS visit our web sile at Www. EDAX.com/integration or call 1.201.529.4880

\section{Visit us at M \& M Booth 416}
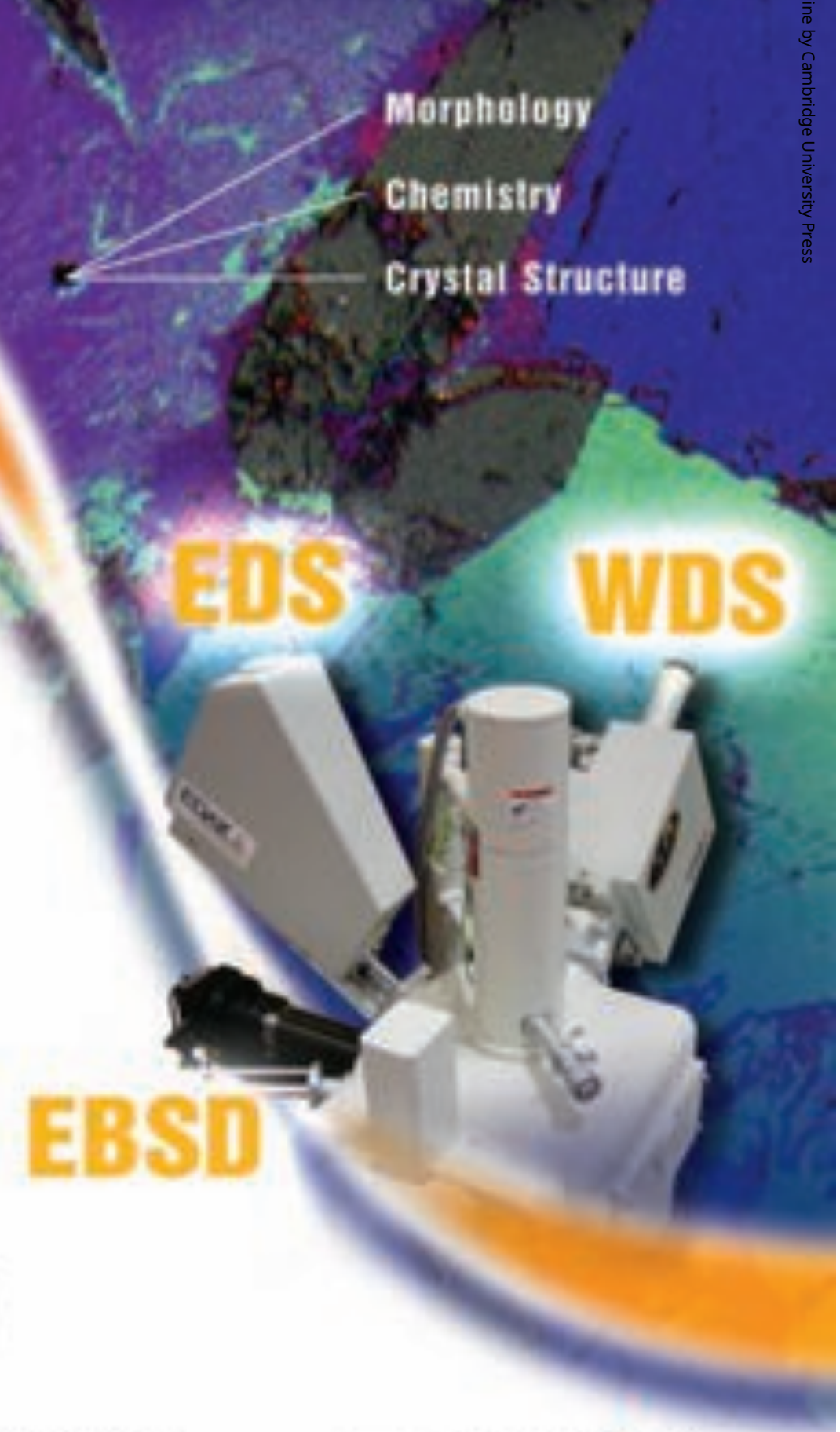

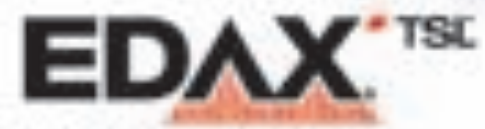

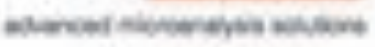

NMETEK 


\section{The ALIS Gas Field Ionization Source Explained}

The ALIS Gas Field Ionization Source differs from the FIM principally in the shape of the tip. The tip shape has been manipulated so that there is a pyramid shaped bump on the end (figure 4). The pyramid edges and apex are atomically sharp. The advantage of this geometry is that the first few ionization discs (at the tip of the pyramid) begin emitting at a relatively low voltage while all the other atoms are not yet capable of emitting. As such, all of the arriving helium gas is shared by just a few atoms (figure 5) instead of a few hundred atoms. Under normal operating conditions the emission from a single atom is selected with an aperture. This permits the beam to have $\sim 100 \times$ the beam current relative to the low current "beamlets" from the FIM.

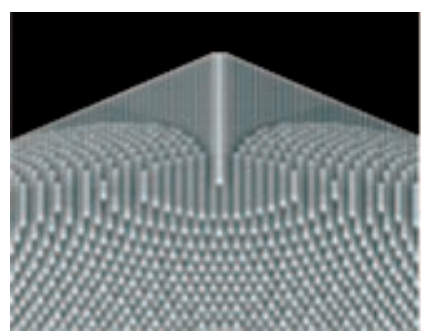

Figure 4: The ALIS source is comprised of a metal tip $(R$ $\sim 100 \mathrm{~nm})$ with an atomically upon it. precise pyramid assembled

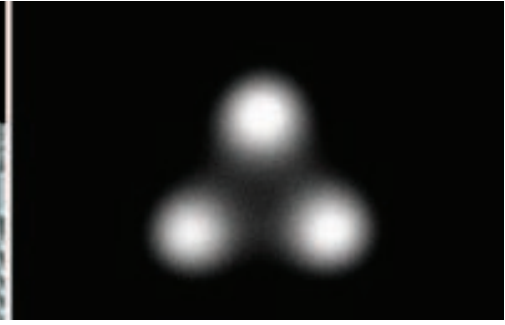

Figure 5: The emission pattern from an ALIS ion source consists of a small number of beams - each of which originates from an atom near the top of the pyramid.
This pyramid can be readily removed by increasing the field to $5 \mathrm{~V} / \AA$ until all pyramid atoms are removed. Subsequently, the pyramid can be rebuilt, and then removed numerous times by a proprietary process.

\section{Advantages of the ALIS Gas Field Ionization Source}

One advantage of the ALIS source is that the beam current can be modulated by simply changing the background pressure of the imaging gas. This can be controlled over several orders of magnitude without any need to change the beam energy, extraction field, or beam steering. Under typical conditions, the beam current from a single atom is $10 \mathrm{pA}$, but operation from $1 \mathrm{fA}$ to $100 \mathrm{pA}$ is practical.

The energy spread of an ALIS ion source is less than $1.0 \mathrm{eV}$ (FWHM). The energy spread arises from the finite thickness of the ionization disc in conjunction with the very high electric field $\sim 3 \mathrm{~V} / \AA$ throughout the disc. Measurements by the authors have established an upper bound of $1.0 \mathrm{eV}$, which is limited by the quality of our spectrometer. This suggests that the ionization disc is approximately $0.3 \AA$ thick or less. Other measurements [2] have indicated that the energy spread is $\sim 0.41 \mathrm{eV}$. In comparison, this energy spread is a factor of $10 \times$ smaller than a liquid metal ion source (LMIS) [1].

The virtual source size of the ALIS ion source is remarkably small. Each ionization region is smaller than the atom spacing, as evidenced by the non-overlapping ionization discs (figure 5). The virtual source size, constructed by back-projecting the ion trajectories once they have left the extraction area, is expected to be considerably smaller than this. With a conservative estimate of the virtual source size of $\sim 3 \AA$, the brightness can be calculated to be $\sim 1.4 \times 10^{9} \mathrm{~A} / \mathrm{cm}^{2} \mathrm{sr}$. This is a factor of $\sim 30 \times$ better than a Schottky electron source, and a factor of $\sim 500 \times$ better than LMIS [3].

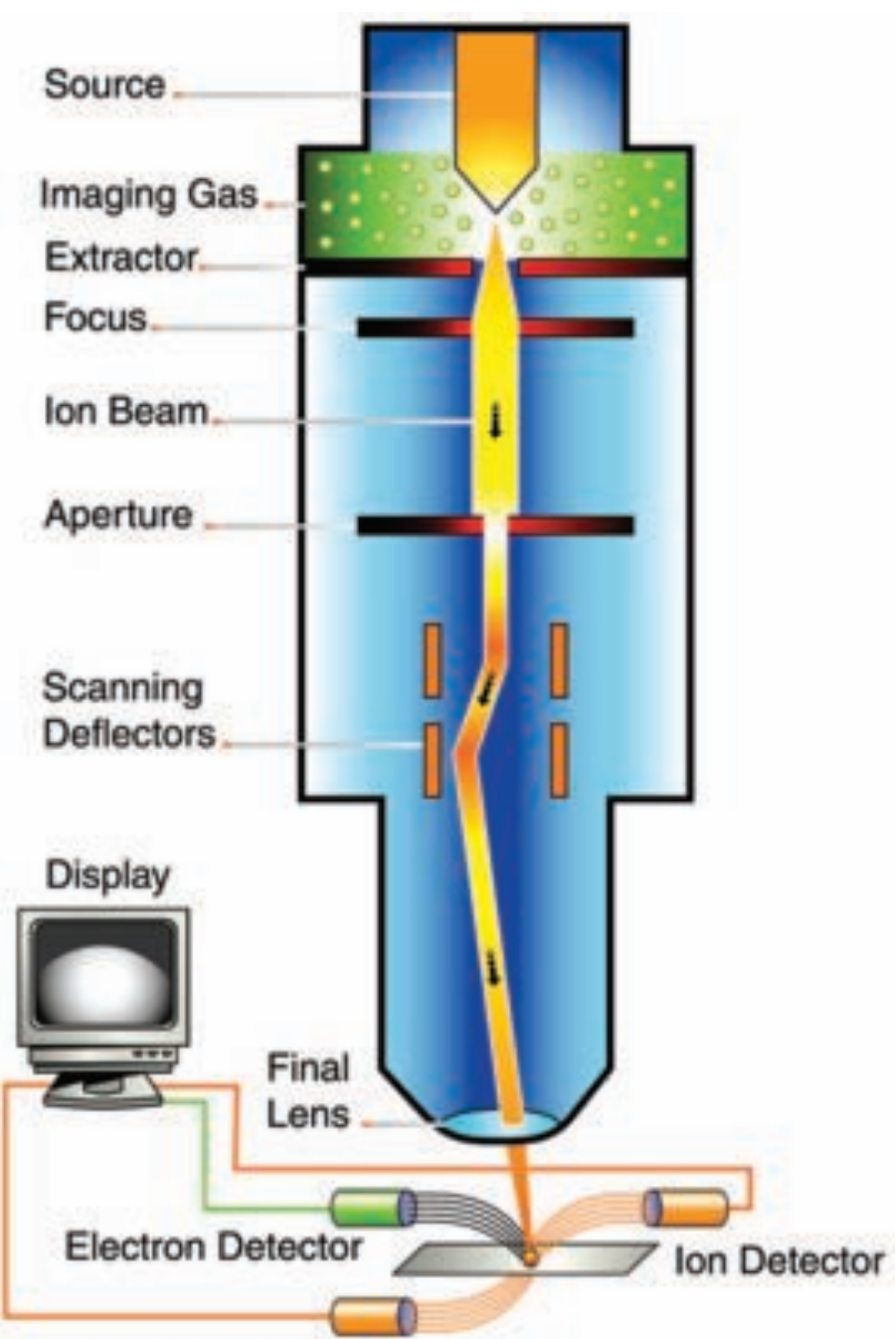

Transmission Detector

Figure 6: ALIS LookingGlass LG-2 imaging system.

Finally, the diffraction effects of a helium ion beam are considerably more favorable than those of an electron beam. A typical electron in a low voltage SEM has a momentum which is small enough that diffraction effects have to be accounted for. In comparison, the ion in a helium ion microscope has a momentum which is $300 \times$ larger. And correspondingly, the De Broglie wavelength is $300 \times$ smaller for the ion under typical operating conditions.

In summary, the ALIS ion source has an energy spread, brightness, and wavelength that are well-suited to producing a high quality beam that can be focused to a sub-nm probe size - even under practical imaging conditions.

\section{Substrate Interaction}

Producing a small beam is the first step in achieving excellent resolution. The next important criterion is to have a small interaction volume in the substrate.

The first plot on the left in figure 7 shows a typical Monte Carlo plot for a gallium ion beam at $30 \mathrm{keV}$ (a typical operating voltage for a FIB). An ion beam from a liquid metal ion source can be focused to a probe size of approximately $5 \mathrm{~nm}$ in the best practical case. However when these relatively heavy ions interact with the substrate, they create a great many sputtered particles and generate many secondary electrons from recoils rather than from the incident ions. This creates an interaction volume that is relatively large. The 


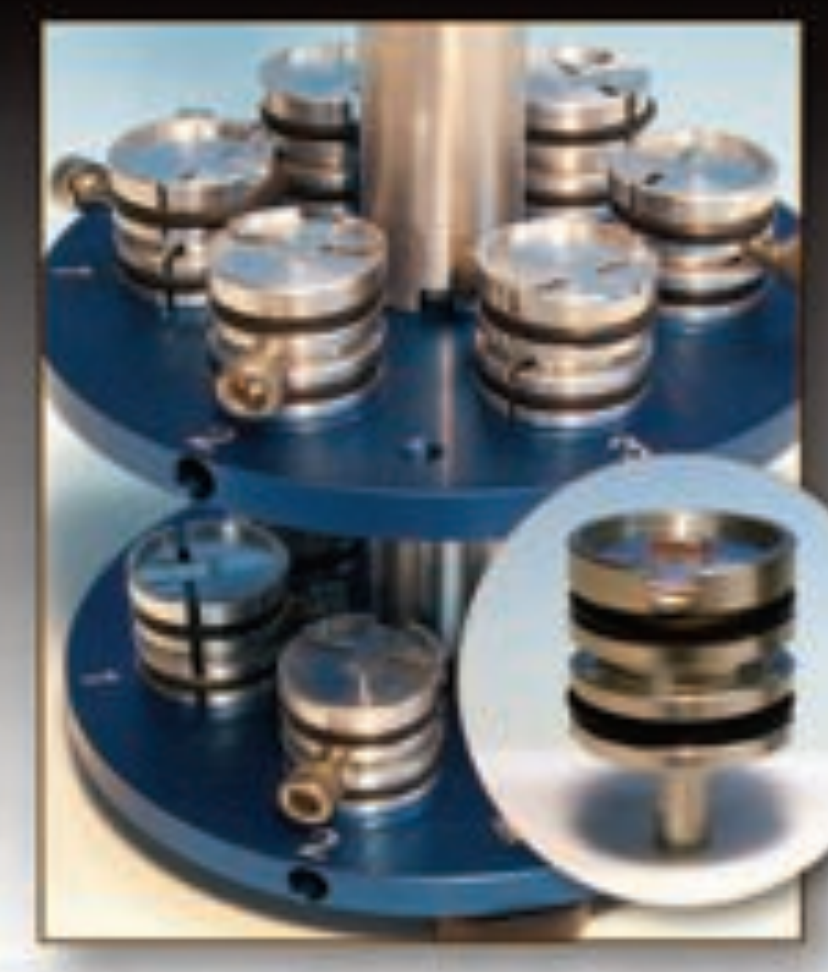

\section{Fortress $^{\text {TM }}$ FIB Holder with CastleGuard ${ }^{\text {IM }}$ Protection}

Fortress $^{\text {TM }}$ FIB Holders are reusable holders that secure FIB samples that are held in a specific orientation without the use of adhesives, adhesive pads, or conductive paint. Fortress ${ }^{\mathrm{TM}}$ FIB Holders can be used to position a thin, whole or cut TEM grid/disk in an orientation such that either an in-situ or an ex-situ FIB lift-out technique can be used to attach a FIB-prepared sample. Physical protection of the mounted sample on the grid is provided with the CastleGuardim protection design. CastleGuard'M protection provides a rigid support structure that shields the sample while allowing required access for processing. Fortress ${ }^{T M}$ FIB Holders are designed to fit into the SS200 SampleSaver ${ }^{\text {TM }}$ storage container for storage and transport in an inert environment. Up to 30 Fortress $^{\mathrm{TM}}$ FIB holders can be stored in a single SS200 SampleSaver ${ }^{r M}$ storage container.

\section{Special Features:}

$>$ Unique clamping mechanism secures sample without over tightening.

$>$ Standard $1 / 8$ " pin mount fits most FIBS and SEMs.

$>$ Peripheral support structure protects specimens during handling and transport.

$>$ Economical design allows user to prepare and store samples on the same holder.
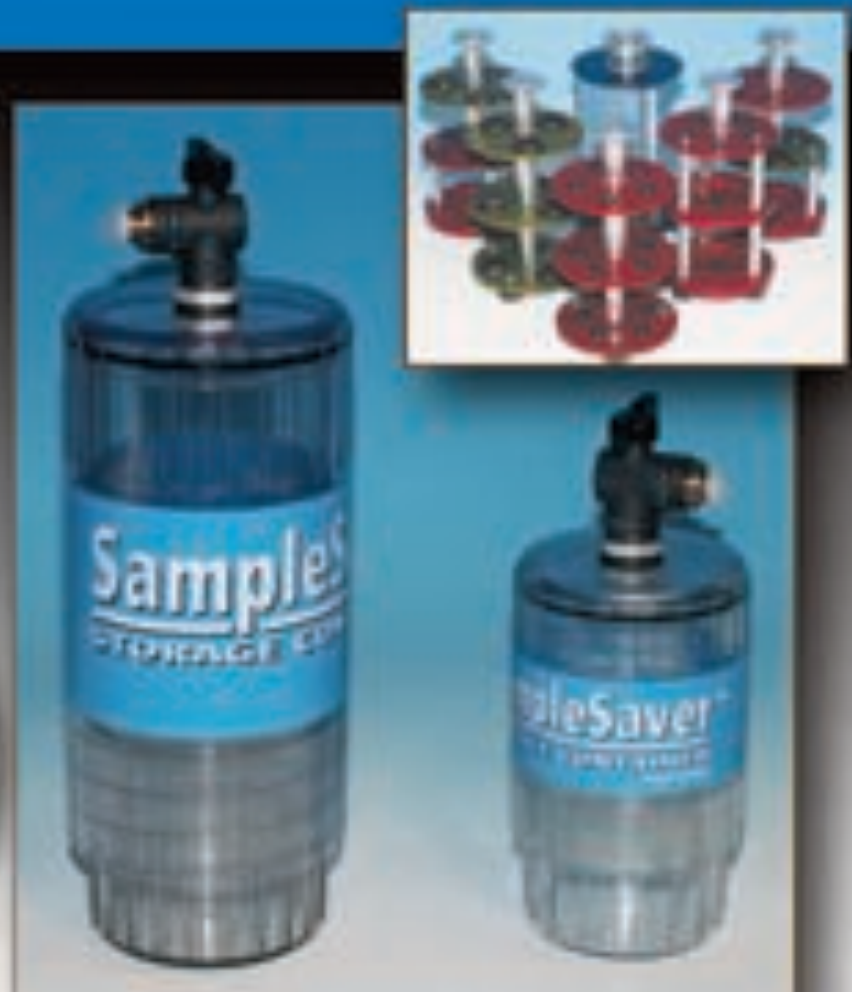

\section{SampleSaver ${ }^{\text {M }}$ Storage Container}

The SampleSaverim Portable Storage Container system is a specialized container that enables a user to replace the reactive atmospheric air typically present in the container with an inert gas species. Once a sample is stored inside the inert atmosphere, the sample does not undergo reactions such as oxidation during storage and/or transportation. It is ideally suited to the preservation of microscopy samples that may undergo changes during the delay between sample preparation and analysis.

\section{Special Features:}

Suitable for SEM, TEM, SPM, EBSD, AFM and FIB samples as well as Liquid Chromatography Vials

$>$ Prevents oxidation of samples and coatings

Sample racks for holding SEM stubs, customized TEM grid boxes, AFM discs, Microscope Slides, LC Vials, FIB Holders and Metallographic mounts

$\checkmark$ Samples remain secure during transport

$>$ Preserves chromium coated SEM samples

$>$ Simple leak check feature to verify integrity

Sample racks are interchangeable and color coded 


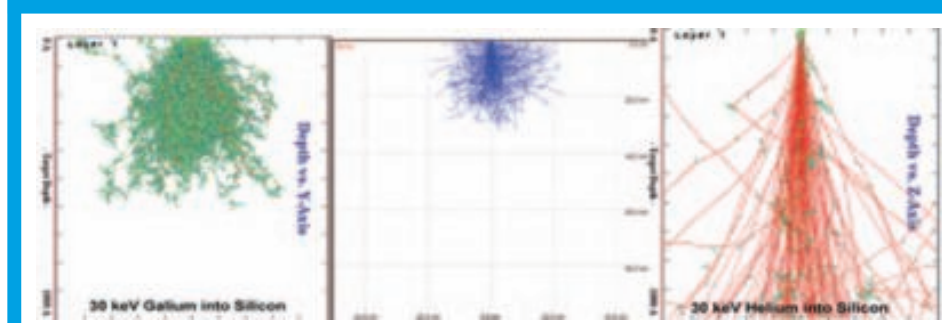

Figure 7: Interaction volume of $30 \mathrm{keV}$ Gallium (left), $1 \mathrm{keV} \mathrm{SEM}$ (center), $30 \mathrm{keV}$ Helium (right).

detectable secondary electrons are generated anywhere in which this volume intersects with the surface. Thus, the resolution in the image is not nearly as small as the spot size.

The center plot shows the condition for a typical SEM. In this case the beam energy is $1 \mathrm{keV}$ into silicon. The lateral straggle of the electrons near the surface is quite large. Collisions in the substrate create many high energy backscattered electrons, which then proceed to create additional secondary electrons near the surface. This cascade of electron production over a fairly large volume limits the resolution capability of scanning electron microscopes.

The plot on the right shows a typical operating condition for the helium ion microscope. This shows a $30 \mathrm{keV}$ beam on silicon. Since the energy is much higher than the energy of the electrons in a SEM (under typical operating conditions) the helium ions penetrate much more deeply into the sample with far less lateral straggle. Since the helium ions are 17 times lighter than the gallium ions in the first example, they do not have a significant recoil contribution. These two benefits allow for a very small interaction volume in the substrate. This small interaction volume insures that the secondary electrons emitted at or near to the surface come from a very small area - much smaller than a SEM or a traditional gallium FIB tool.

\section{Secondary Electron Yield}

The secondary electron yield produced by the helium ion beam is quite high and varies considerably from one material to another. An experiment was conducted to quantify the secondary electron yield from a variety of metals. In this experiment, the helium ion beam passed through a grid that was $98 \%$ transparent to the beam. This grid could be biased from $-30 \mathrm{~V}$ to $+30 \mathrm{~V}$ in order to control the secondary electrons. A Faraday cup on the sample stage was used to measure the current of the ion beam. The entire stage was ungrounded so that the current induced by the secondary electrons leaving the sample could be measured for different samples. The experimental set up is described in figure 8 .

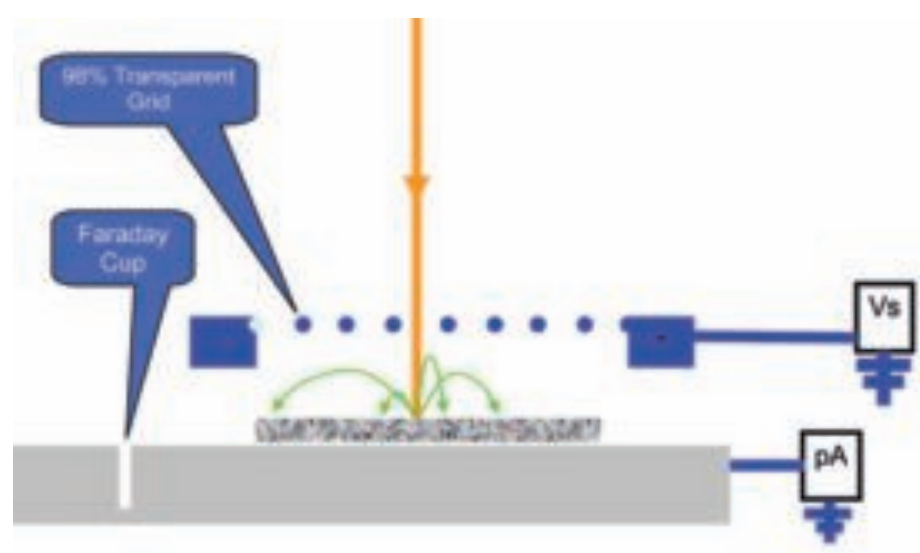

Figure 8: Experimental set up for secondary electron yield characterization

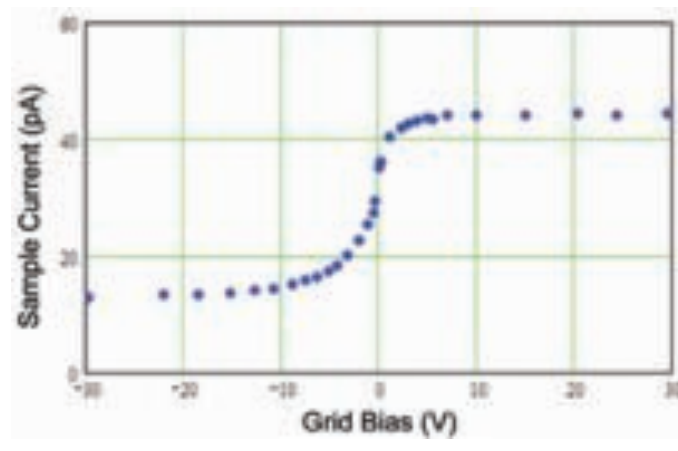

Figure 9: Sample current versus grid bias for silicon under a $20 \mathrm{keV}$ Helium ion beam.

As e ach metal sample was tested, the voltage to the transparent grid was swept from -30 Volts to +30 Volts as the current from the stage was monitored. With the grid potential set to -30 Volts,

none of the secondary electrons can escape and they are all directed back to the surface. In this condition, the current measured on the stage was exactly equal to the beam current measured in the Faraday cup - $13 \mathrm{pA}$. This implies that there are no sputtered positive ions. If there were, the two current readings would be different.

As the grid voltage is swept through zero, the stage current changes dramatically as the secondary electrons are allowed to escape, thus creating a current on the stage in addition to the current from the incident helium ion beam. In this way, the secondary electron yield of different materials can be measured.

Table 1 shows the secondary electron yields measured for a variety of metals.

\begin{tabular}{|c|c|c|c|}
\hline Material & $Z$ & $\mathbf{M}(\mathbf{a m u})$ & YSE $(\boldsymbol{E p}=\mathbf{2 0} \mathbf{k e V}, \boldsymbol{\alpha p}=\mathbf{0})$ \\
\hline Aluminum & 13 & 27.0 & 4.31 \\
\hline Silicon & 14 & 28.1 & 2.38 \\
\hline Titanium & 22 & 47.9 & 3.65 \\
\hline Iron & 26 & 55.8 & 3.55 \\
\hline Nickel & 28 & 58.7 & 4.14 \\
\hline Copper & 29 & 63.4 & 3.23 \\
\hline Indium & 49 & 114.8 & 4.69 \\
\hline Tungsten & 74 & 183.8 & 2.69 \\
\hline Rhenium & 75 & 186.2 & 2.61 \\
\hline Platinum & 78 & 195.1 & 7.85 \\
\hline Gold & 79 & 197.0 & 4.17 \\
\hline Lead & 82 & 207.2 & 4.57 \\
\hline
\end{tabular}

As can be seen from the table, the secondary electron yield varies widely from one material to another - from 2-8 secondary electrons for each incoming helium ion. This very high secondary electron yield allows for good signal to noise in the image. The wide range of values provides material contrast far superior to that which can be attained using secondary electrons in a SEM.

An example of this advantageous secondary electron yield can be seen by comparing figures 10 and 11 . Figure 10 is a SEM image of an alignment cross. It is a very good SEM image and has excellent resolution. It does however lack material contrast. The image in figure 11 is a secondary electron image taken in the ALIS tool. In this image it is quite clear that the material in the center of the alignment cross is different from the material on the outside of the cross. One can also see small dark areas of contamination on the 


\section{thinkforward}

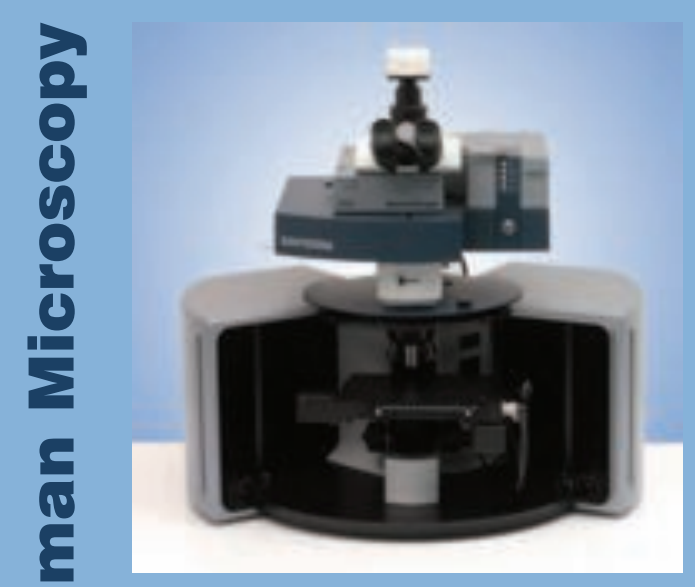

$\square$ All-in-one, compact, confocal design

口 Multiple wavelegths; $1064 \mathrm{~nm}$, $785 \mathrm{~nm}, 532 \mathrm{~nm}$ and/or $633 \mathrm{~nm}$.

$\square$ Sure_Cal ${ }^{\circledast}$ automatic continuous calibration

$\square$ Spectral imaging utilizing sample stage mapping

$\square$ Confocal depth profiling with FlexFocus ${ }^{\mathrm{TM}}$

$\square$ Automatic fluorescence rejection using SERDS for $785 \mathrm{~nm}$

\section{Isn't it time to make peace between two technologies?}

\section{Introducing the SENTERRA ${ }^{\text {TM }}$}

Dispersive Raman Microscope with the new integrated 1064nm FT-Raman technology

Bruker Optics introduces the first Raman microscope system to combine the long wavelength benefits of $1064 \mathrm{~nm}$ excitation with the scattering benefits of shorter wavelength Raman excitation. The new 'hybrid' platform accommodates the RamanScope fluorescence-free Fourier transform Raman system and the SENTERRA grating based dispersive Raman technology with fluorescence rejection tools. This combination provides full spectroscopic characterization and optimizes the strengths of the both techniques for your complex micro-analysis samples.
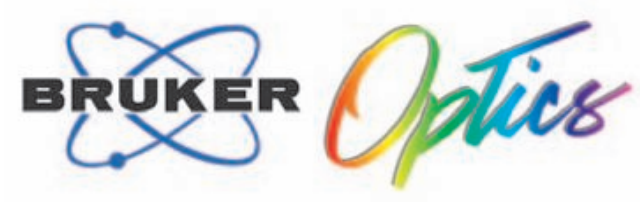

for more information please visit: www.brukeroptics.com/microscopy 1-888-4BRUKER | microscopy@brukeroptics.com 

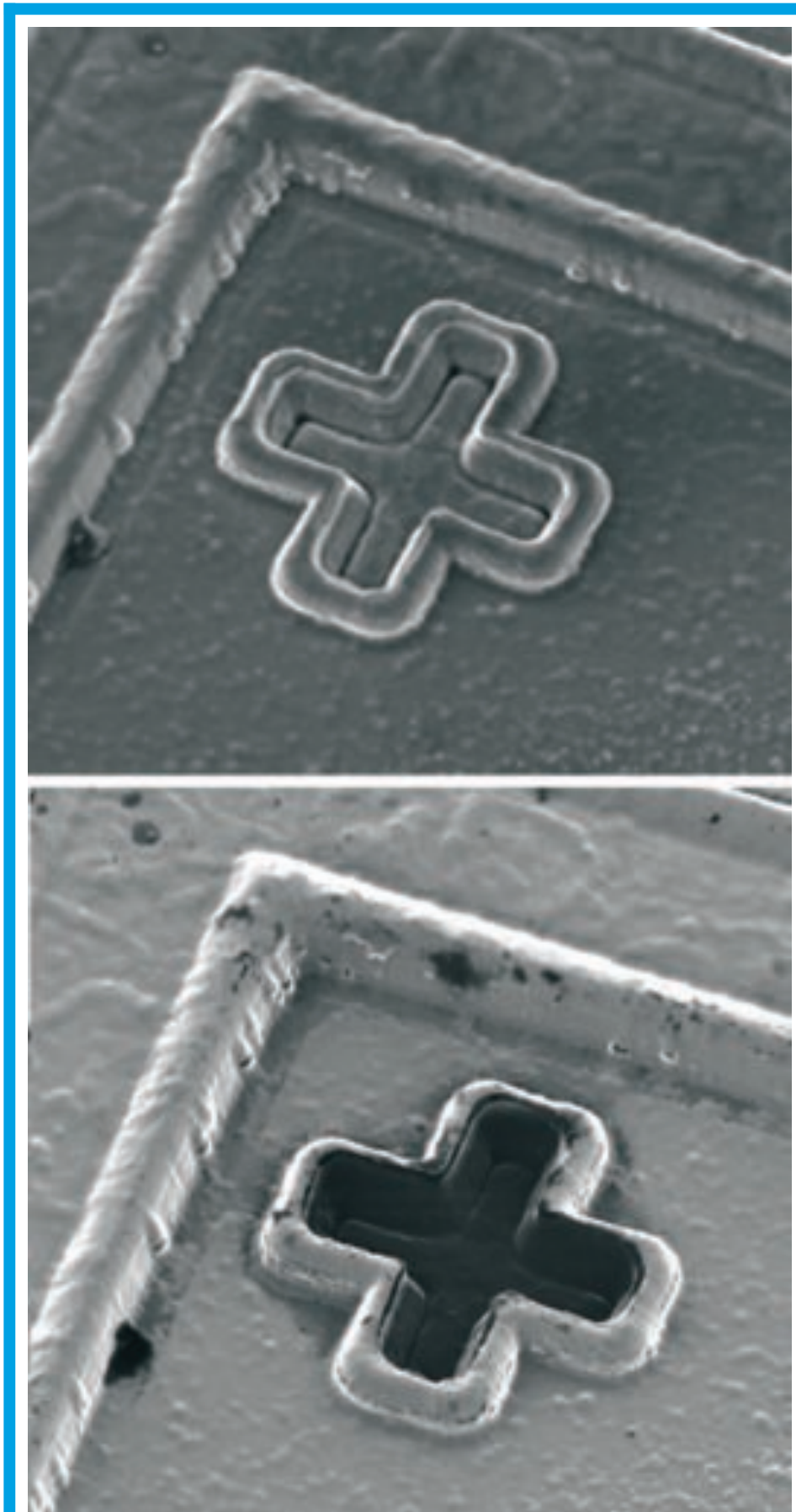

Figure 10: (top) Secondary electron SEM image of alignment cross.

Figure 11: (bottom) Secondary electron ALIS image of same alignment cross

sidewalls outside of the cross that are not readily apparent in the SEM image.

\section{RBI Imaging}

While the helium ions do not cause any discernable sputtering of the surface, the incident ions do backscatter from the substrate when they collide with heavier ions. This is similar to the ion backscattering found in Rutherford Backscattered Spectroscopy. As the helium ions collide with other, heavier atoms, they transfer some of their momentum to the target atoms. The energy of the backscattered helium ions is determined by the atomic mass of the target nucleus and the angle of the scattering. The heavier the target nucleus, the more energetic is the backscattered helium ion.

The number of backscattered helium ions is proportional to $\sim Z^{2}$ of the target nucleus. With a properly designed detector, a

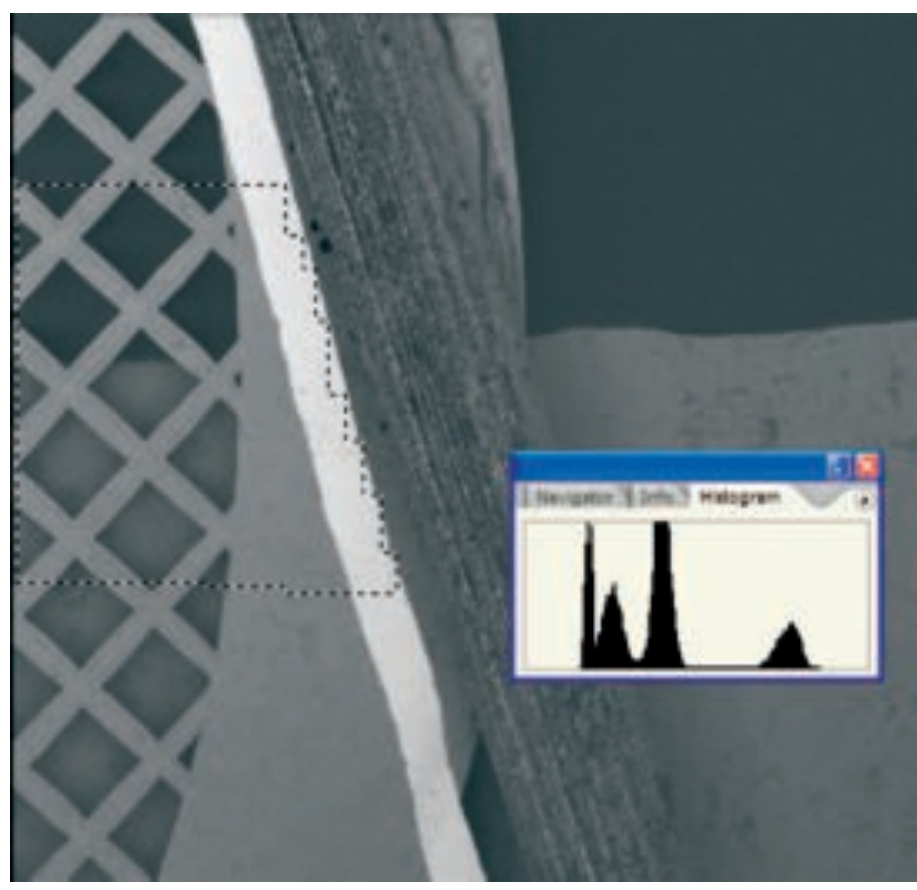

Figure 12: RBI image of grid, clearly showing 4 distinct peaks in the image histogram corresponding to carbon, nickel, copper and gold.

qualitative assessment can be made of the $\mathrm{Z}$ of the target nuclei. This is a quick and easy way to discriminate between materials while maintaining excellent spatial resolution. An example is shown in figure 12.

The distinct peaks in the histogram correspond to the different materials in the target. From lowest intensity to highest, these are carbon, nickel, copper and gold.

This imaging mode offers rich material information that may be missing from a secondary electron image. Figure 13 shows a secondary electron image of a solder bump. While there is some information in the image, mostly from topography, it is not possible to tell the tin and lead apart in this image. Figure 14 shows the same area imaged in RBI mode. In this image, it is clear which areas are tin and which are lead. The bright areas are tin as this is the higher $Z$ material in this sample.

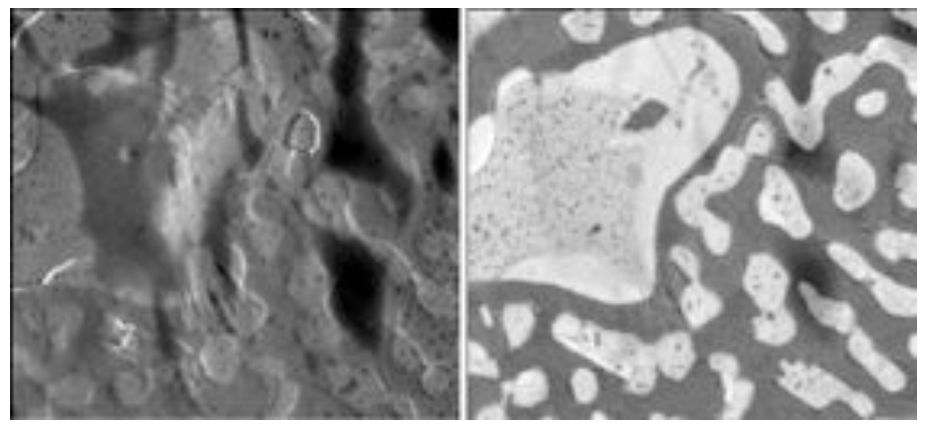

Figure 13: (left) Secondary electron image of a solder bump showing topography, but not much material difference.

Figure 14: (right) RBI image of same solder bump clearly showing the difference between areas of tin (dark) and lead (bright).

Another advantage of RBI mode is that it obviates the need to conductively coat charging samples. Since the backscattered ions are at very high potential, it does not really matter if the sample charges up to a few hundred volts. It does not affect the tools ability to image the sample. Figure 15 shows a benthic foraminifer imaged in RBI mode. This image shows excellent contrast and depth of field on an insulating sample. 


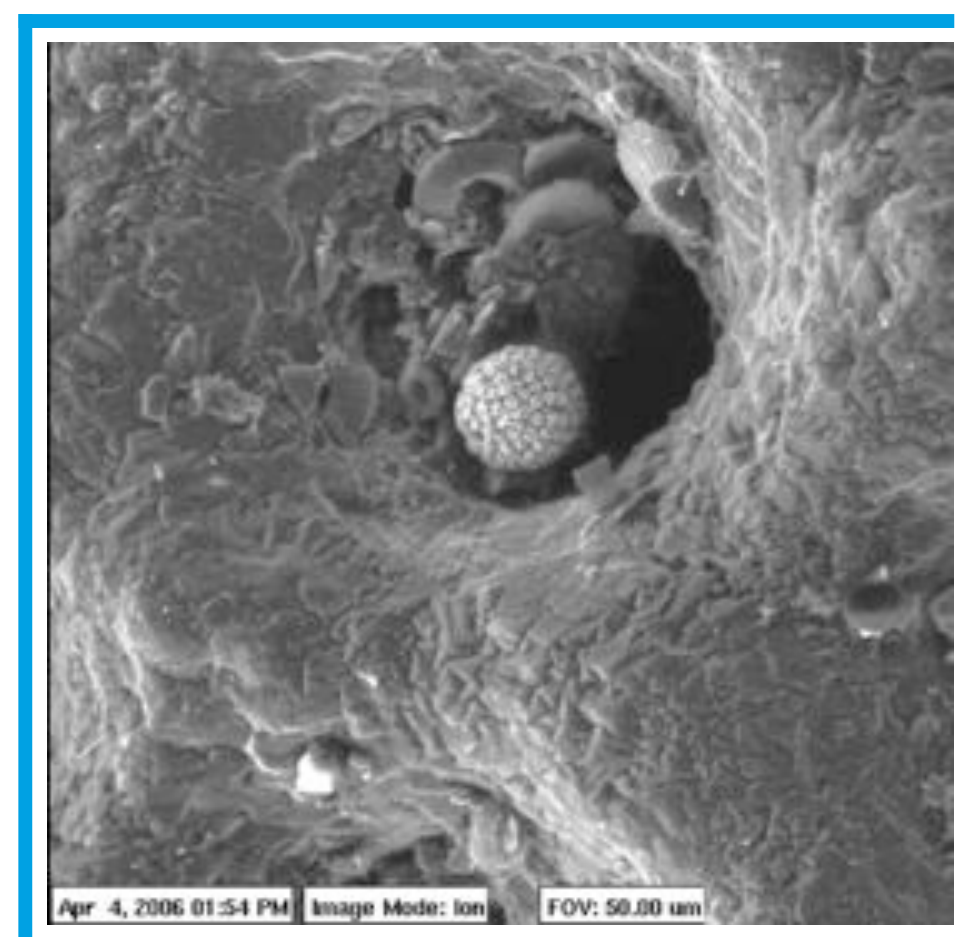

Figure 15 RBI image of benthic foraminifer.

\section{Conclusions}

It has been demonstrated that the helium ion microscope has several unique properties that, when combined together, will allow for higher resolution imaging than that available today with conventional scanning electron microscopes. In addition to better resolution, the helium ion microscope also provides unique contrast mechanisms both in secondary electron mode and RBI mode that enable material discrimination and identification.

\section{References}

[1] V. N. Tondare, "Quest for High Brightness, Monochromatic Noble Gas Ion Source," J. Vac. Sci. Technol. A, Vol23, No. 6, p.1498-1507 (2005).

[2] N. Ernst, G. Bozdech, H. Schmidt, W. A. Schmidt, Grover L. Larkins. "On the Full-Width-at-Half-Maximum of Field Ion Energy Distributions, "Applied Surface Science 67, p.111-117 (1993).

[3] Jon Orloff (editor), "Handbook of Charged Particle Optics," CRC Press, (1997).

\section{ERRATA}

In the article in the May 2006 issue:

\section{Precise SEM Cross Section Polishing via Argon Beam Milling}

$$
\text { by }
$$

N. Erdman, R. Campbell, and S. Asahina

The correct caption for figure 7 on page 25 should be:

Figure 7. Image of a yeast cell cross section.

This was communicated to me by the authors prior to printing the issue and I failed to make the correction.

\section{Failure is not an option.}

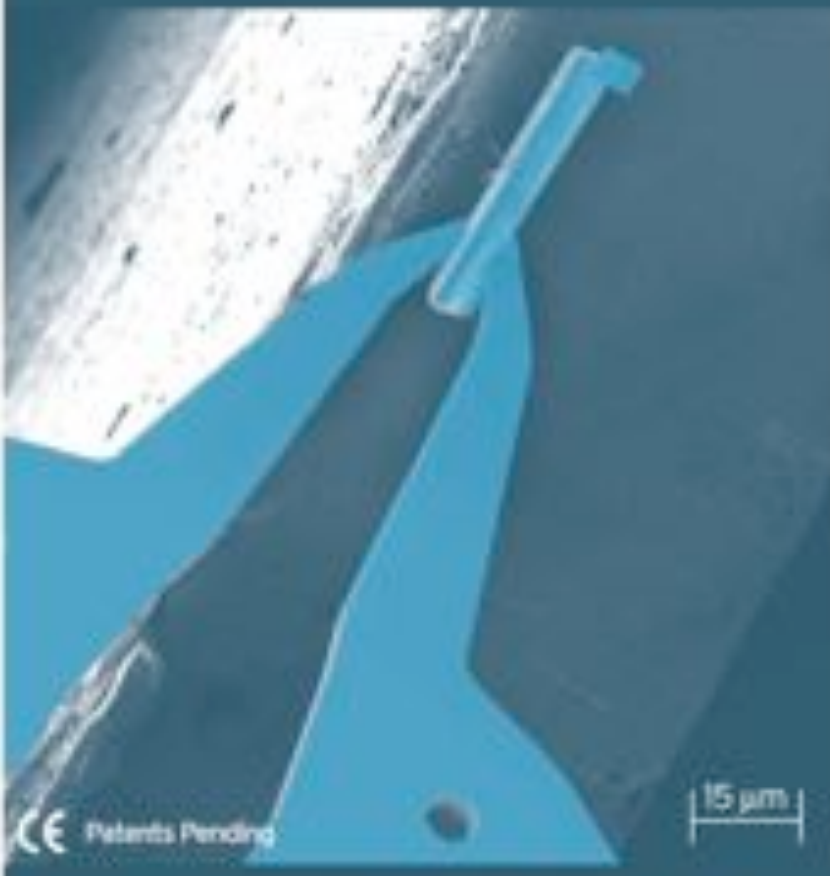

Whac yesre performing TEM Samcle Prep. you don't have time to waste.

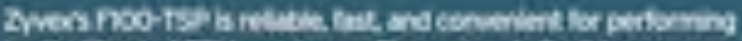

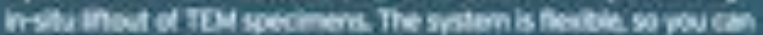

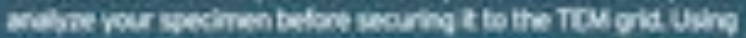

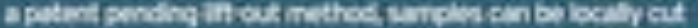

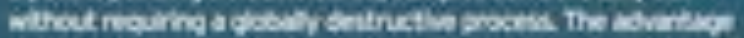

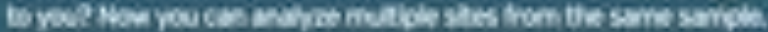

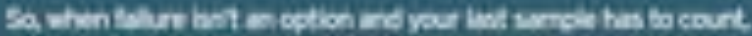

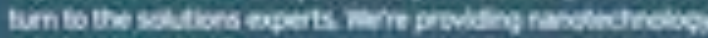

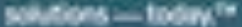

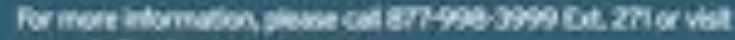
or wiosite at wiskmesen

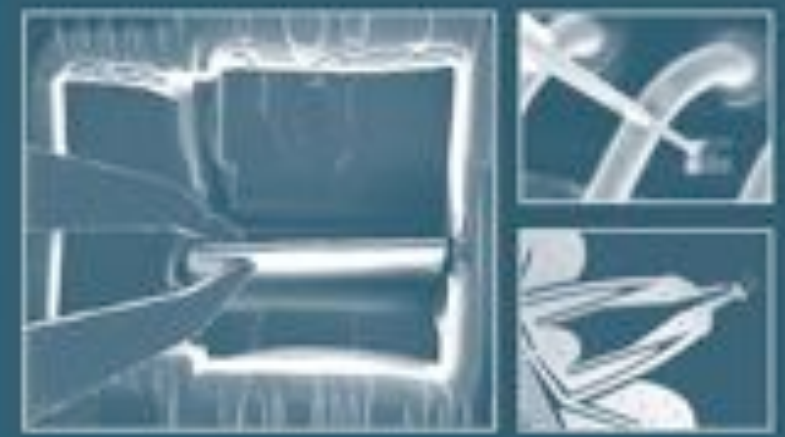

